\title{
Hydrophilic Silica/Copolymer Nanoparticles and Protein-Resistance Coatings
}

\author{
Hongpu Huang1, Ling $\mathrm{He}^{2^{*}}$ \\ ${ }^{1}$ Xi'an Jiaotong University, School of Science, Department of Chemistry, Xi'an, 710049, China \\ ${ }^{2}$ Department of Chemistry, School of Science, Xi'an Jiaotong University, Xianning West Road, 28, Xi'an, \\ 710049 China \\ Email: *heling@mail.xjtu.edu.cn
}

Received 13 November 2015; accepted 5 January 2016; published 11 January 2016

\begin{abstract}
Hydrophilic silica/copolymer nanoparticles of $\mathrm{SiO}_{2}$-g-P(PEGMA)-b-P(PEG) are prepared by silica surface-initiating atom transfer radical polymerization (SI-ATRP) of poly (ethylene glycol) methyl ether methacrylate (PEGMA) and poly(ethylene glycol) methacrylate (PEG), by using Three molar ratios of $\mathrm{SiO}_{2}$-Br/PEGMA/PEG as $1 / 42.46 / 19.44,1 / 42.46 / 38.88$ and $1 / 42.46 / 77.76$. Their temperature sensitive behaviour, $\mathrm{pH}$ response and surface properties as protein-resistance coatings are characterized. $220 \mathrm{~nm}$ core-shell nanoparticles as P(PEGMA)-b-P(PEG) shell grafted on $\mathrm{SiO}_{2}$ core are formed in water solution, which gained LCST at $60^{\circ} \mathrm{C}-77^{\circ} \mathrm{C}$ and good dispersion in water when $\mathrm{pH}>$ 5.0. The water-casted films by $\mathrm{SiO}_{2}$-g-P(PEGMA)-b-P(PEG) obtain a little rough surface $(\mathrm{Ra}=26.8$ - $29.7 \mathrm{~nm})$. While, the introduction of $P(P E G)$ segments could slight increase the protein-repelling adsorption of $\mathrm{SiO}_{2}$-g-P(PEGMA)-b-P(PEG) films $(\Delta \mathrm{f}=-6.96 \mathrm{~Hz} \sim-7.25 \mathrm{~Hz})$ compared with $\mathrm{SiO}_{2}$-g-P(PEGMA) films $(\triangle \mathrm{f}=-\mathbf{9} .5 \mathrm{~Hz})$. Therefore, SiO2-g-P(PEGMA)-b-P(PEG) could be used as protein-resistance coatings.
\end{abstract}

\section{Keywords}

Silica/Copolymer, Hydrophilic Nanoparticles, Tem-Responsive, Protein-Resistance, Coatings

\section{Introduction}

Hydrophiphilic block copolymers are mostly used in biological sciences [1] [2] and advance material [3]. In the recent research, the significant hydrophiphilic block copolymers are mainly used poly (poly (ethylene glycol) methyl ether methacrylate, P (PEGMA), or poly (poly (ethylene glycol) methacrylate, P(PEG), as the hydrophilic block [4]-[7]. Actually, both P(PEGMA) and P(PEG) consist of a linear methacrylate backbone reactive functional group with a side chain of poly(ethylene glycol) (PEG), which could generate a wealth of new polymeric materials by employing the recently developed living radical polymerization techniques of reversible addition fragmentation chain transfer (RAFT) [8] [9] and atom transfer radical polymerization (ATRP) [10]-[12]. In order to achieve the polymer films with adjusted properties by varying the monomer composition based on

${ }^{*}$ Corresponding author.

How to cite this paper: Huang, H.P. and He, L. (2016) Hydrophilic Silica/Copolymer Nanoparticles and Protein-Resistance Coatings. Journal of Materials Science and Chemical Engineering, 4, 18-23. http://dx.doi.org/10.4236/msce.2016.41004 
PEGMA monomer, well-defined block copolymers are synthesized, such as P(PEGMA)-b-poly(2,5-dibromo 3-vinylthiophene) by RAFT for uniform cross-linked nanoparticles [8], poly (glycidyl methacrylate-co-poly (ethylene glycol) methyl ether methacrylate) nanoparticles by ATRP [12]. For investing the effect of concentration and temperature on the micelle formation by $\mathrm{P}$ (PEGMA) in aqueous solution, the P(PEGMA) homopolymersare synthesized by aqueous ATRP [13]. Furthermore, The PEG-based materials are commonly used as biofouling-resistant materials. Thus, many hydrophobic surfaces modified with PEG-based materials are greatly used to reduce protein adsorption [14]-[16]. Actually, the distribution density and the chain length of PEG grafted on the surfaces are two key parameters in determining protein repelling behaviour [17]. Therefore, it is hoped that a hydrophilic block copolymers as antibiofouling coating could be designed with the combination of two different hydrophilic segments.

This paper reports the synthesis, the tem-responsive and surface properties as protein-resistance coatings of the hydrophiphilic silica graftedblock copolymers of $\mathrm{SiO}_{2}$-g-P(PEGMA)-b-P(PEG) nanoparticles, which are synthesized via SI-ATRP approach by poly [poly (ethylene glycol) methyl ether methacrylate)] and (P(PEGMA)) poly (poly (ethylene glycol) methacrylate, $\mathrm{P}(\mathrm{PEG})$ ) in three molar ratios of $\mathrm{SiO}_{2}$-Br/PEGMA/12FMA = $1 / 42.46 / 19.44,1 / 42.46 / 38.88$ and $1 / 42.46 / 77.76$. Their chemical structures are characterized by nuclear magnetic resonance $\left({ }^{1} \mathrm{H}-\mathrm{NMR}\right)$ spectroscopy, Fourier transform infrared spectroscopy (FI-IR) and thermo gravimetric analysis (TGA). The nanoparticles in water are characterized by transmission electron microscopy (TEM). The transition points and phase separation processes for the lower critical solution temperature (LCST) and pH-Response are examined by dynamic light scattering (DLS). The surface properties of films are investigated by atomic force microscope (AFM) and the quartz crystal microbalance with dissipation (QCM-D). The obtained results provide a prospected insight on the application of $\mathrm{SiO}_{2}$-g-P(PEGMA)-b-P(12FMA) nanoparticles as protein-resistance coatings.

\section{Experimental}

\subsection{Materials}

$\mathrm{SiO}_{2}$-initiator $\left(\mathrm{SiO}_{2}\right.$ - $\left.\mathrm{Br}\right)$ was prepared by silanization of hydrosilylated undec-10-enyl, 2-bromo-2-methyl propionate (UBMP) and silica nanoparticle using the previous method [18]. Poly (ethylene glycol) methyl ether methacrylate (PEGMA, $\sim 475 \mathrm{~g} \cdot \mathrm{mol}^{-1}$ ) and poly (ethylene glycol) methacrylate (PEG, $\sim 500 \mathrm{~g} \cdot \mathrm{mol}^{-1}$ ) was supplied Aldrich. $\mathrm{CuCl}$ and $\mathrm{CuCl}_{2}$ were purified. $\mathrm{N}, \mathrm{N}, \mathrm{N}$, N,' N"-pentamethyldiethylenetriamine (PMDETA), ethanol, tetrahydrofuran (THF) and other solvents were used as analytical purity. Bovine serum albumin (BSA) was prepared in distilled water and phosphate buffer solution (PBS) buffer solution.

\subsection{Synthesis of $\mathrm{SiO}_{2}-\mathrm{g}-\mathrm{P}(\mathrm{PEGMA})-\mathrm{b}-\mathrm{P}(\mathrm{PEG})$ by SI-ATRP}

The $\mathrm{SiO}_{2}$-g-P(PEGMA)-b-P(PEG) are prepared by silica surface-initiating atom transfer radical polymerization (SI-ATRP) of poly (ethylene glycol) methyl ether methacrylate (PEGMA) and poly(ethylene glycol) methacrylate (PEG). The procedure of $\mathrm{SiO}_{2}$-g-P(PEGMA)-b-P(PEG) polymerization was similar in previous work [19]. The conversion of PEGMA was $82 \%$ and the conversion of PEG was $80 \%$ after $12 \mathrm{~h}$ reaction. In this paper, three molar ratios of $\mathrm{SiO}_{2}$-Br/PEGMA/PEG=1/42.46/19.44, 1/42.46/38.88 and 1/42.46/77.76 for Sample S1, S2 and $\mathrm{S} 3$ were used to obtain $\mathrm{SiO}_{2}$-g-P(PEGMA)-b-P(PEG) hybrid particles. The results from ${ }^{1} \mathrm{H}-\mathrm{NMR}, \mathrm{FI}-\mathrm{IR}$ and TGA were able to confirm that the synthesis of $\mathrm{SiO}_{2}$-g-P(PEGMA)-b-P(PEG)was proceed as expected by SIATRP approach.

\section{Characterization}

The chemical structure, morphology of nanoparticles, transition points and phase separation processes for the lower critical solution temperature (LCST), surface properties of films and protein-resistance behavior was invested and the detail information was showed in previous work [19].

\section{Results and discussion}

\subsection{The Morphology of Hydrophiphilic Nanoparticles}

Because the nanoparticles formed by hydrophilic block copolymer are strongly depended on the composition of 
blocks, the influence of different molar ratios of $\mathrm{SiO}_{2}$-Br/P(PEGMA)/P(PEG) in Sample S1 (1/42.46/19.44), S2 (1/42.46/38.88) and S3 (1/42.46/77.76) on the morphology of $\mathrm{SiO}_{2}$-g-P(PEGMA)-b-P(PEG) nanoparticles in water is explored by TEM (Figure 1), based on the good solubility of hydrophilic block of P(PEGMA) and $\mathrm{P}(\mathrm{PEG})$ in water. In Figures 1(a)-(c), the spherical particles composed of $\sim 220 \mathrm{~nm}$ silica core and different thickness of P(PEGMA) shell are observed for $\mathrm{SiO}_{2}$-g-P(PEGMA)-b-P(PEG) of Sample S1-S3. All the spherical particles have a good dispersion in water. While, the thickness of -P(PEGMA)-b-P(PEG) shell is increased with increasing the PEGMA concentration from Sample S1 to Sample S3, but much content of P(PEGMA) segment in Sample S3 leads to the overlapping cross of copolymer (Figure 1(c)).

\subsection{The Tem-Responsive of LCST in Water Solution}

The lower critical solutiontemperature (LCST) and the transition points and phase separation behavior of $\mathrm{SiO}_{2}-$ P(PEGMA)-b-P(PEG) nanoparticles in dilute aqueous solutionis determinedby DLS measurementsas the concentration of $1.0 \mathrm{mg} \cdot \mathrm{ml}^{-1}$. The LSCT is taken as the initial turn point in the hydrodynamic diameter $\left(\mathrm{D}_{\mathrm{h}}\right)$ versus temperature. The variation of $\mathrm{D}_{\mathrm{h}}$ in Figure 2(a) exhibits an abrupt increase at the transition point for $\mathrm{SiO}_{2}-$ $\mathrm{P}(\mathrm{PEGMA})-\mathrm{b}-\mathrm{P}(\mathrm{PEG})$ with only one transition point during heating. It is noticed that the LCSTfor Sample S1 and Sample S2of $\mathrm{SiO}_{2}-\mathrm{P}(\mathrm{PEGMA})-\mathrm{b}-\mathrm{P}(\mathrm{PEG})$ is at $60^{\circ} \mathrm{C}$ and $67^{\circ} \mathrm{C}$, which is much lower than the LCST of the $\mathrm{SiO}_{2}-\mathrm{P}(\mathrm{PEGMA})\left(80^{\circ} \mathrm{C}\right)$ [19]. This is because the grafting $\mathrm{P}(\mathrm{PEG})$ segment onto $\mathrm{SiO}_{2}-\mathrm{P}(\mathrm{PEGMA})$ enhances the intermolecular forceand the intermolecular force is enhancedwith the increasing content of $\mathrm{P}(\mathrm{PEG})$. the enhanced intermolecular forcecoulddrops the LCST of $\mathrm{SiO}_{2}$-P(PEGMA). Sample S3 has the less content of $\mathrm{P}(\mathrm{PEG})$ segment to reduce the effect of intermolecular force to obtain a high LCST at $77^{\circ} \mathrm{C}$. This indicates that the effect of intermolecular force is gradually enhanced with the increasing of hydrophilic block of P(PEG). Thus, the interaction of $\mathrm{SiO}_{2}$-g-P(PEGMA)-b-P(PEG) nanoparticles is increased and the interaction between water and -P(PEGMA)-b-P(PEG) is reduced from Sample S1 to S3, which makes the LCST of $\mathrm{SiO}_{2}$-gP(PEGMA)-b-P(PEG)reduce gradually.

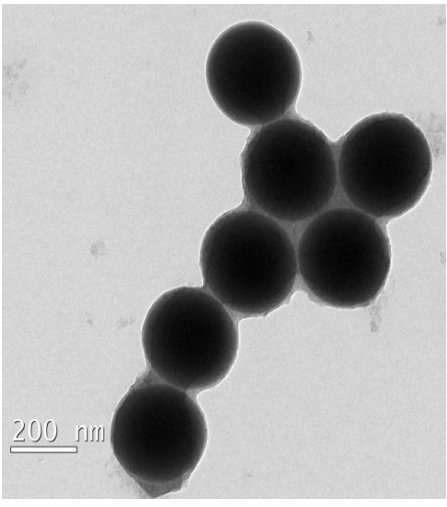

(a)

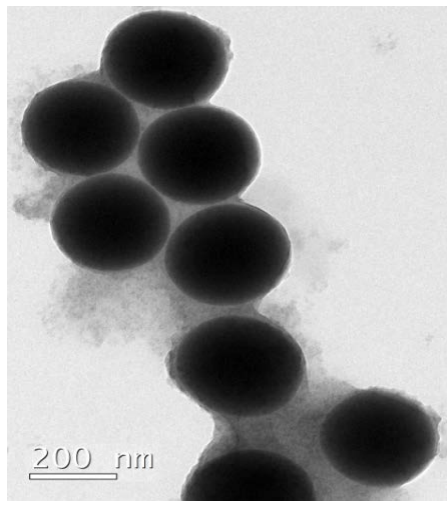

(b)

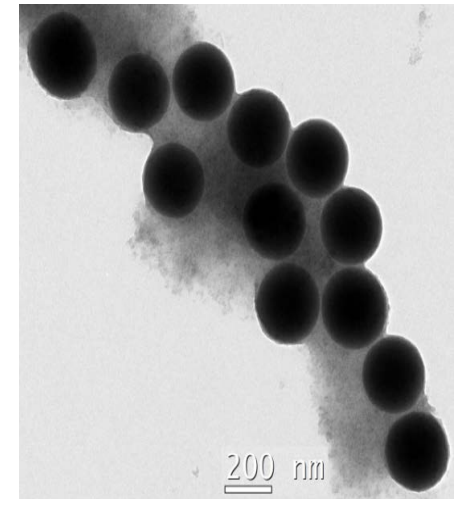

(c)

Figure 1. TEM images of $\mathrm{SiO}_{2}$-g-P(PEGMA)-b-P(PEG) in water for $\mathrm{S} 1$ (a), $\mathrm{S} 2$ (b) and $\mathrm{S} 3$ (c).
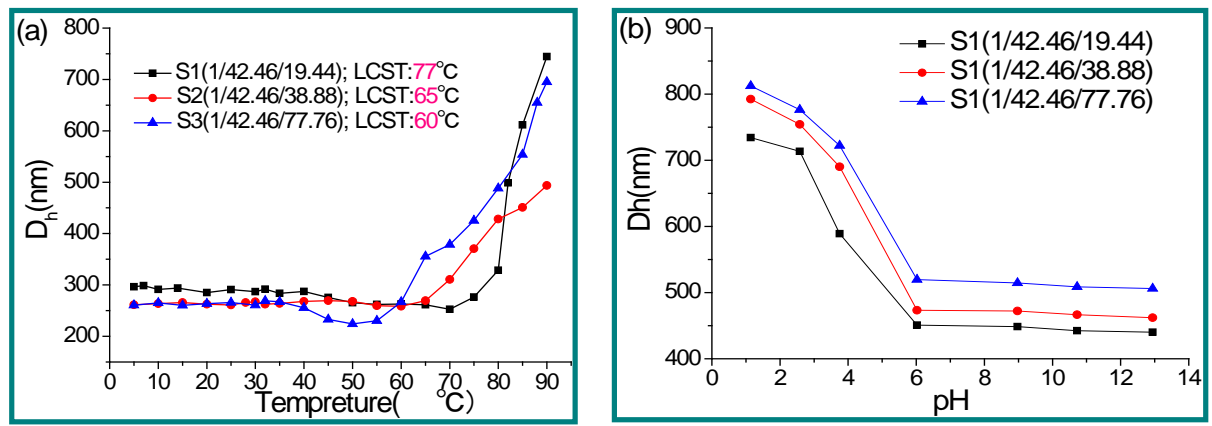

Figure 2. DLS measurements of LCST for Sample S1-S3 in water (a) and Dependence of hydrodynamic diameter (Dh) of $\mathrm{SiO}_{2}$-g-P(PEGMA)-b-P(PEG)on $\mathrm{pH}(\mathrm{b})$ at the concentration of $0.2 \mathrm{mg} \cdot \mathrm{ml}^{-1}$. 
Actually, the pH-Responsive self-assembly behavior of $\mathrm{SiO}_{2}$-g-P(PEGMA)-b-P(PEG) was also analyzed by DLS. The Dh of $\mathrm{SiO}_{2}$-g-P(PEGMA)-b-P(PEG) in water is decreased with the ascending of $\mathrm{pH}$ in Figure 2(b). The hydration is weakened and the intermolecular forces is reinforced due to the deprotonation degree of P(PEG) segment rose with increasing acidity of aqueous solution. This result leads the $\mathrm{pH}$ is decreased with the ascending of $\mathrm{pH}$ increased. When $\mathrm{pH}$ is above 5.0, the Dh remains essentially unchanged with the ascending of $\mathrm{pH}$. This is due to the hydroxyl of P(PEG) segment combines with the hydroxyl of aqueous solution and this hindered the intermolecular aggregates. So the $\mathrm{SiO}_{2}$-g-P(PEGMA)-b-P(PEG) particles have a good dispersion when $\mathrm{pH}$ is above 5.0.

\subsection{The Chemical Composition, Morphology and Water Adsorption of Films}

The chemical compositions of $\mathrm{SiO}_{2}$-g-P(PEGMA)-b-P(PEG) particles are analyzed by XPS in Figure 3 . The particles for S1 (Figure 3(a)), S2 (Figure 3(b)) and S3 (Figure 3(c)) are mainly composed of O, C and Si elements and the electron binding energy of O1s, C1s and Si2p at $530.1 \mathrm{eV}, 283.0 \mathrm{eV}$ and $101.2 \mathrm{eV}$, respectively. With the increasing amount of $\mathrm{P}(\mathrm{PEG})$ segment, the single of $\mathrm{Si}$ is gradually weakened and the single of $\mathrm{C}$ is increased. This has been proved by the chemical composition in Figures 3(a)-(c).

The morphology of film surface for $\mathrm{SiO}_{2}$-g-P(PEGMA)-b-P(PEG) nanoparticles casted from water solution is investigated by AFM (Figures 3(d)-(f)). For three $\mathrm{SiO}_{2}$-g-P(PEGMA)-b-P(PEG) films, due to the decreasing of silica content and the increasing of the P(PEG) segment, the root mean square roughness (Ra) for Sample S1, S2 and S3 is slightly reduced as Ra $=29.7 \mathrm{~nm}, 28.1 \mathrm{~nm}$ and $26.8 \mathrm{~nm}$ for (Figures 3(d)-(f)), respectively. They are all distributed with particle raised agglomerates and the particle raised agglomerates are reduced from Sample S1 to S3. Therefore, with the increase of P(PEG) segment, the surface roughness of films is decreased in water.

Because the chemical composition and surface roughness contribute much to the surface water adsorption of $\mathrm{SiO}_{2}$-g-P(PEGMA)-P(PEG) films monitored by QCM-D in Figure 4(a). The $\Delta f$ in the adsorption curves is used to indicate the adsorbed amounts of probe liquids, the $\Delta D$ is used to indicate the viscoelasticity of the film (the higher value of $\Delta D$ indicating the higher viscoelasticity of the film). In Figure 4(a), the similar absorption curves for water-casted films by $\mathrm{SiO}_{2}$-g-P(PEGMA)-b-P(PEG) (Sample S1, S2 and S3)indicate that $\Delta f$ and $\Delta D$

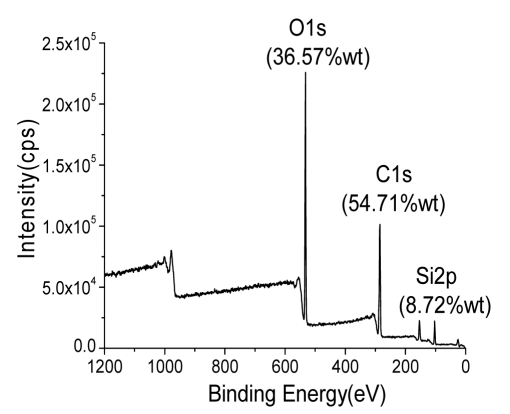

(a)

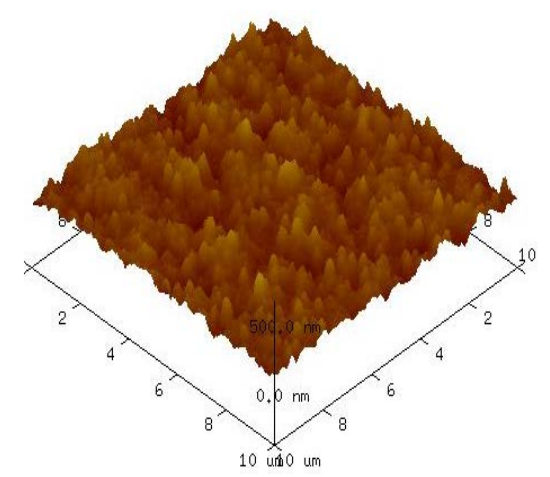

(d)

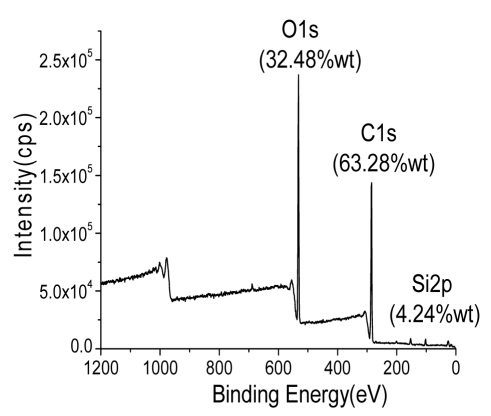

(b)

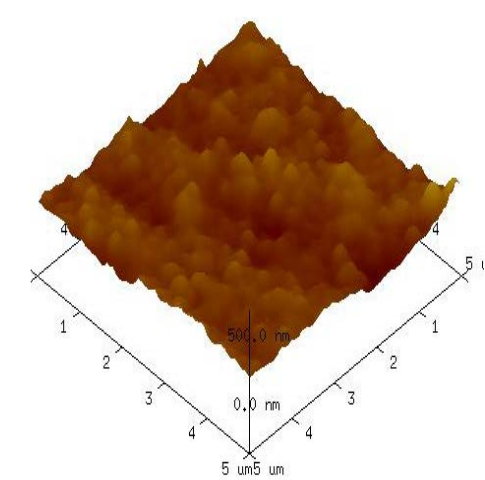

(e)

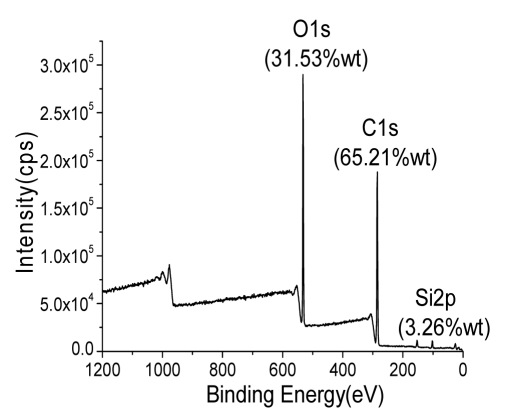

(c)

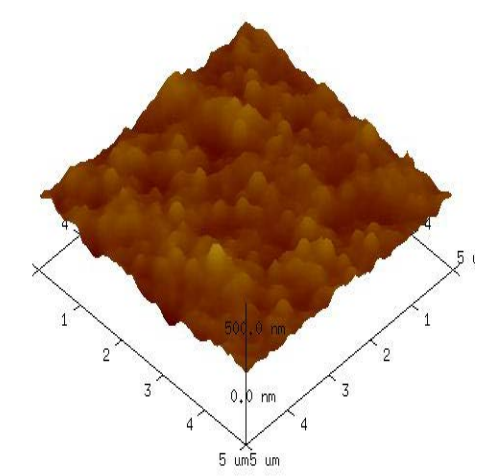

(f)

Figure 3. XPS scanning spectrum of the powder $\mathrm{SiO}_{2}$-g-P(PEGMA)-b-P(PEG) for S1 (a); S2(b) and S3 (c); and AFM images of water-casted films of S1 Ra $=29.7 \mathrm{~nm}(\mathrm{~d})$; S2 Ra $=28.1 \mathrm{~nm}$ (e); S3 Ra = $26.8 \mathrm{~nm}$ (f). 

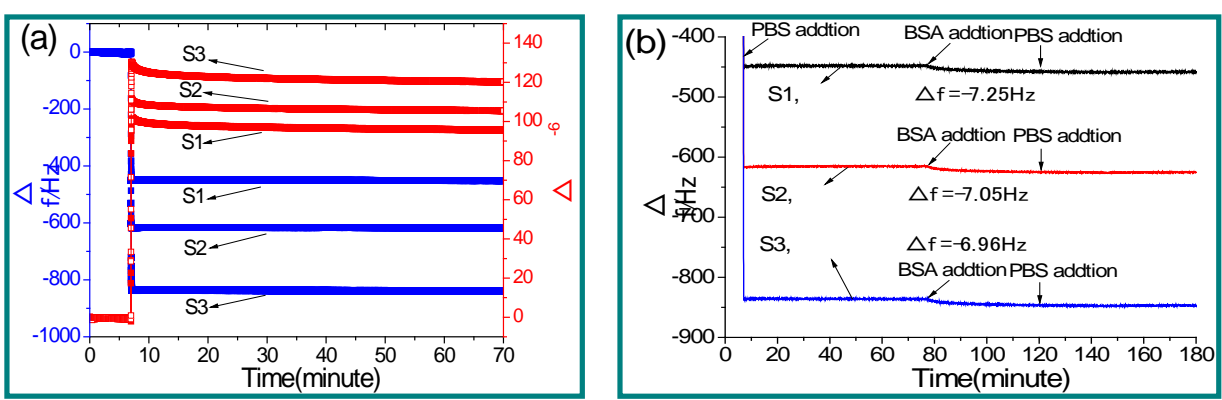

Figure 4. QCM-D curves on the surface of Sample S1, S2 and S3water-casted films (a) and Proteinresistance behavior on the surface of $\mathrm{SiO}_{2}$-g-P(PEGMA)-b-P(PEG) films (Sample S1, S2 and S3) (b).

quickly reach the adsorption equilibrium when water is absorbed on the surface of films, indicating that the $\mathrm{P}$ (PEGMA) and P(PEG) chains are distributed on the surface in a relatively ordered structure. The adsorbed amount of water is increased from $\mathrm{S} 1$ to $\mathrm{S} 3(\Delta f=-447.67,-615.48$ and $-836.11 \mathrm{~Hz}$, respectively) due to the increasing of the $\mathrm{P}(\mathrm{PEG})$ content. At the same time, the viscoelasticity of films is correspondingly increased $\left(\Delta D=96.51,106.23\right.$ and $\left.121.12 \times 10^{-6}\right)$. These indicates the increasing $\mathrm{P}(\mathrm{PEG})$ chains in water results the increase of the water adsorption amount and decrease of the surface roughness (Figures 3(d)-(f)).

\subsection{The Application to Protein-Resistance}

It is well known that PEG-modified surfaces are normally resisted to protein adsorption [20], and therefore are used to demonstrate the ability of antibiofouling. The less adsorption of BSA demonstrates the better antibiofouling of film. To correlate the relationship between the wettability and the anti-bacteria of obtained$\mathrm{SiO}_{2}$-g-P(PEGMA)-b-P(PEG), the BSA protein-resistance of the copolymer films is investigated by using the method of QCM-D (Figure 4(b)). The S1-S3 films show just a slightly increase in $\Delta f(-7.25 \mathrm{~Hz},-7.15 \mathrm{~Hz}$ and $-6.96 \mathrm{~Hz}$, respectively) after the adsorption of BSA (Figure 4(b)), compared with PBS adsorption. When the PBS solution is continued flowing on the surface about $120 \mathrm{~min}$, the $\Delta f$ almost have no changes to suggest a very little adsorbed amount of BSA protein on the film surfaces. This indicates that Samples S1-S3 have good protein resistance due to their hydrophilic character and sterical effects of PEG chains. The introduction of $\mathrm{P}(\mathrm{PEG})$ segments could slight increase the protein-repelling adsorption of $\mathrm{SiO}_{2}$-g-P(PEGMA)-b-P(PEG) films $(\Delta f=-6.96 \mathrm{~Hz} \sim-7.25 \mathrm{~Hz})$ compared with $\mathrm{SiO}_{2}$-g-P(PEGMA) films $(\Delta f=-9.5 \mathrm{~Hz})$ [19]. This is attributed to the well-distributed hydrophilic PEG chains on the film surfaces to give a repellent property to protein adsorption.

\section{Acknowledgements}

This work has been financially supported by the National Natural Science Foundation of China (NSFC Grants No. 51373133, 51573145 and 51073126), by the National Basic Research Program of China (973 Program, No.2012CB720904), by the International Cooperation Project of Shaanxi Province (No.2014KW11) and the State Administration of Cultural Heritage (20110128). The authors also wish to express their gratitude for the MOE Key Laboratory for Non-equilibrium Condensed Matter and Quantum Engineering of Xi'an Jiaotong University.

\section{References}

[1] Mustafa, B., Burak, Z.B., Mustafa, S.Y. and Mehmet, V.Y. (2015) Smart-Polymer-Functionalized Graphene Nanodevices for Thermo-Switch-Controlled Biodetection. Biomater. Sci. Eng, 1, 27-36. http://dx.doi.org/10.1021/ab500029h

[2] Philippe, H.S., Bradley, A., Bruce, M.A. and Jeffrey, P.Y. (2007) Synergistic Activity of Hydrophilic Modification in Antibiotic Polymers. Biomacromolecules, 8, 19-23. http://dx.doi.org/10.1021/bm0605513

[3] Sehmus, O., Liehui, G., Tharangattu, N.N., Amelia, H.C.H., Hyunseung, Y., Srividya, S., Robert, V. and Pulickel, M.A. (2014) Anisotropically Functionalized Carbon Nanotube Array Based Hygroscopic Scaffolds. Appl. Mater. Interfaces, 6, 10608-10613. http://dx.doi.org/10.1021/am5022717 
[4] Liu, Y.G., Qiu, Q., Shen, W.Q. and An, Z.S. (2011) Aqueous Dispersion Polymerization of 2-Methoxyethyl Acrylate for the Synthesis of Biocompatible Nanoparticles Using a Hydrophilic RAFT Polymer and a Redox Initiator. Macromolecules, 44, 5237-5245. http://dx.doi.org/10.1021/ma200984h

[5] Oana, G.S., Georges, M.P., Hannes, P.E., Michael, A.R.M., Richard, H. and Ulrich, S.S. (2009)A Versatile Approach to Unimolecular Water-Soluble Carriers: ATRP of PEGMA with Hydrophobic Star-Shaped Polymeric Core Molecules as an Alternative for PEGylation. Macromolecules, 42, 1808-1816. http://dx.doi.org/10.1021/ma8024738

[6] Zhu, X.B., Michael, F., Benjamin, T.D., Marc, A.I. and Bradford, B.W. (2012) Modifying the Hydrophilic-Hydrophobic Interface of PEG-b-PCL to Increase Micelle Stability: Preparation of PEG-b-PBO-b-PCL Triblock Copolymers, Micelle Formation, and Hydrolysis Kinetics. Macromolecules, 45, 660-665. http://dx.doi.org/10.1021/ma202530v

[7] Torben, G., Canet, A., Lucio, I., Schlu, D.A., Nicholas, D.S. and Marcus, T. (2013) PEG-Stabilized Core-Shell Nanoparticles: Impact of Linear versus Dendritic Polymer Shell Architecture on Colloidal Properties and the Reversibility of Temperature-Induced Aggregation. Nano, 7, 316-329.

[8] Nakabayashi, K., Oya, H. and Mori, H. (2012) Cross-Linked Core-Shell Nanoparticles Based on Amphiphilic Block Copolymers by RAFT Polymerization and Palladium-Catalyzed Suzuki Coupling Reaction. Macromolecules, 45, 3197-3204. http://dx.doi.org/10.1021/ma300239u

[9] Cao, C.W., Yang, K., Wu, F., Wei, X.Q., Lu, L.C. and Cai, Y.L. (2010) Thermally Induced Swellability and Acid-Liable Dynamic Properties of Microgels of Copolymers Based on PEGMA and Aldehyde-Functionalized Monomer. Macromolecules, 43, 9511-9521. http://dx.doi.org/10.1021/ma1017549

[10] Huang, C., Koon, G.N. and En, T.K. (2012) Combined ATRP and “Click” Chemistry for Designing Stable TumorTargeting Superparamagnetic Iron Oxide Nanoparticles. Langmuir, 28, 563-571. http://dx.doi.org/10.1021/la202441j

[11] Guo, W.H., Zhu, J., Cheng, Z.P., Zhang, Z.B. and Zhu, X.L. (2011) Anticoagulant Surface of 316 L Stainless Steel Modified by Surface-Initiated Atom Transfer Radical Polymerization. Appl. Mater. Interfaces, 3, 1675-1680. http://dx.doi.org/10.1021/am200215x

[12] Liu, J.L., He, W.W., Zhang, L.F., Zhang, Z.B., Zhu, J. and Yuan, L. (2011) Bifunctional Nanoparticles with Fluorescence and Magnetism via Surface-Initiated AGET ATRP Mediated by an Iron Catalyst. Langmuir, 27, 12684-12692. http://dx.doi.org/10.1021/la202749v

[13] Hazrat, H., Khine, Y.M. and Chaobin, H. (2008) Self-Assembly of Brush-Like Poly[poly(ethylene glycol) methyl ethermethacrylate] Synthesized via Aqueous Atom Transfer Radical Polymerization. Langmuir, 24, 13279-13286. http://dx.doi.org/10.1021/la802734e

[14] Chen, X., Zhang, G.F., Zhang, H.Q., Zhan, X.L. and Chen, F.Q. (2015) Preparation and Performance of Amphiphilic Polyurethane Copolymers with Capsaicin-Mimic and PEG Moieties for Protein Resistance and Antibacteria. Ind. Eng. Chem. Res, 54, 3813-3820. http://dx.doi.org/10.1021/ie505062a

[15] Kim, D.G., Kang, H., Han, S. and Lee, J.C. (2012) Dual Effective Organic/Inorganic Hybrid Star-Shaped Polymer Coatings on Ultrafiltration Membrane for Bio- and Oil-Fouling Resistance. Appl. Mater. Interfaces, 4, 5898-5906. http://dx.doi.org/10.1021/am301538h

[16] Wetra, Y., Sophie, M., Pierre, T.M., Maureen, C.E., James, C.A., Lyndsey, T., Bo, L. and Thomas, E. (2014) Hydration and Chain Entanglement Determines the Optimum Thickness of Poly(HEMA-co-PEG10MA) Brushes for Effective Resistance to Settlement and Adhesion of Marine Fouling Organisms. Appl. Mater. Interfaces, 6, 11448-11458. http://dx.doi.org/10.1021/am502084x

[17] Chang, Y., Shih, Y.J., Ko, Y.C., Jhong, F.J., Liu, Y.L. and Wei, T.C. (2011) Hemocompatibility of Poly(vinylidene fluoride) Membrane Grafted with Network-Like and Brush-Like Antifouling Layer Controlled via Plasma-Induced Surface PEGylation. Langmuir, 27, 5445-5455. http://dx.doi.org/10.1021/la1048369

[18] Huang, H.P. and He, L. (2014) Silica-Diblock Fluoropolymer Hybrids Synthesized by Surface-Initiated Atom Transfer Radical Polymerization. RSC Adv, 4, 13108-13118. http://dx.doi.org/10.1039/c3ra47393g

[19] Huang, H.P., Qu, J. and He, L. (2015) Amphiphilic Silica/Fluoropolymer Nanoparticles: Synthesis,Tem-Responsive and Surface Properties as Protein-Resistance Coatings. Journal of Polymer Science, Part A: Polymer Science. http://dx.doi.org/10.1002/pola.27785

[20] Marlene, L., Andre, M. and Christine, B. (2012) Fouling Release Coatings: A Nontoxic Alternative to Biocidal Antifouling Coatings. Chem. Rev., 112, 4347-4390. http://dx.doi.org/10.1021/cr200350v 